\title{
Control of Interfacial Diels-Alder Reactivity by Tuning the Plasma Polymer Properties
}

\author{
Madeline Vauthier, ${ }^{\dagger,+}$ Loïc Jierry, ${ }^{\S}$ Fouzia Boulmedais, ${ }^{\S}$ Jamerson C. Oliveira, ${ }^{\|, \perp}$ \\ Kathryn F. A. Clancy, ${ }^{\dagger, \dagger}$ Chloé Simet, ${ }^{\dagger, \dagger}$ Vincent Roucoules, ${ }^{\dagger, \dagger}$ and Florence Bally-Le Gall*, ${ }^{\dagger, \dagger}$ \\ ${ }^{\dagger}$ Université de Haute-Alsace, CNRS, IS2M UMR 7361, F-68100 Mulhouse, France \\ ${ }^{\ddagger}$ Université de Strasbourg, F-67081 Strasbourg, France \\ ${ }^{\S}$ Institut Charles Sadron, CNRS, UPR 022, F-67034 Strasbourg, France \\ "Chair of Forest Biomaterials, Faculty of Environment and Natural Resources, University of Freiburg, D-79085 Freiburg, Germany \\ ${ }^{\perp}$ Freiburg Materials Research Center, University of Freiburg, D-79104 Freiburg, Germany
}

Supporting Information

\begin{abstract}
Functionalizing the surface of a material with a smart plasma polymer coating is an interesting alternative strategy to obtain a thermoresponsive material without changing its formulation. On the basis of a low-pressure plasma polymerization process, the present work first aims to fabricate polymer thin films that react via the wellknown thermoreversible Diels-Alder (DA) reaction (diene/dienophile cycloaddition). A two-step surface modification process based on (pulsed) plasma polymerization enables the design of functional coatings that contain furan (diene) groups. The reactivity of these surfaces with maleic anhydride (dienophile) in solution is thoroughly investigated, mainly by studying the kinetics of the DA reaction by advancing contact angle measurements. The determination of rate constants of reactions at various temperatures leads to the quantification of thermodynamic parameters such as the activation energy of the reaction as well as the enthalpy and entropy of activation related to the formation of the transitionstate complex involved in the DA reaction. More interestingly, the design of furanfunctionalized coatings with various physicochemical properties enables the understanding of the role played by the density of functional groups and the cross-linking rate of the polymer on the interfacial reactivity. Thus, we show in this work how to control the interfacial DA reaction on plasma coatings by tailoring the operating conditions of plasma polymerization.
\end{abstract}

\section{INTRODUCTION}

Stimuli-responsive materials have properties that depend on the environment in which they are used. ${ }^{1-3}$ In most cases, the material itself is formulated to react to the corresponding stimulus. However, many phenomena occur at the surface of the material. ${ }^{4}$ Therefore, instead of changing the formulation and the bulk properties of the material, providing stimuliresponsive properties selectively to the surface of the material is an interesting alternative strategy for the development of smart materials. Several surface functionalization methods can be used to modify the outermost layer of a material and to obtain a functional thin film on the material. ${ }^{5,6}$ For instance, physical or chemical surface modification techniques such as the elaboration of self-assembled monolayers (SAMs) ${ }^{7}$ or multilayered materials and ${ }^{8-10}$ grafting of small molecules (silanes, isocyanates, etc.) ${ }^{11,12}$ or polymers ${ }^{13}$ are commonly reported. Recently, several groups have explored the use of bioinspired materials, such as polydopamine derivatives, to functionalize the surface of various materials thanks to catechol groups. ${ }^{14,15}$ To avoid the use of solvent or, more generally, of a wet chemical method during the fabrication of a polymer coating, chemical vapor deposition (CVD) processes have been developed to produce the required chemical groups on the surface of a material. ${ }^{16}$ Plasma-enhanced CVD, also known as plasma polymerization, enables the grafting of a great variety of chemical groups (such as anhydrides, ${ }^{17}$ amines, ${ }^{18}$ aniline groups, ${ }^{19}$ alcohols, ${ }^{20}$ or fluorinated groups ${ }^{21,22}$ ) via the growth of a functional polymer thin film. ${ }^{23}$ Additionally, the physicochemical properties of the deposited polymer film (retention rate of functional groups, film roughness, thickness, and cross-linking) can be finely controlled by the operating conditions of plasma polymerization. ${ }^{18,24-26}$ In particular, pulsed plasma polymerization, which comprises two distinct reaction regimes corresponding to the plasma duty cycle (DC) on and off periods, ${ }^{17,27,28}$ strongly limits precursor fragmentation and ion bombardment compared to continuous plasma polymerization. This process was exploited to increase the retention rate of chemical groups within the plasma polymer and to favor its postmodification. ${ }^{29}$ This surface functionalization technique, including a postmodification step, has been

Received: June 16, 2018

Revised: August 23, 2018

Published: September 1, 2018 
chosen for this study to design polymer coatings that have diene groups, more precisely furan functions, capable of DielsAlder (DA) reaction. The DA reaction is a thermoreversible [4 +2 ] cycloaddition between a diene and a dienophile, ${ }^{30}$ frequently used in materials science. ${ }^{31}$ This reaction was thoroughly studied, mainly between furan derivatives (diene) and maleimide derivatives (dienophile), in solution or in bulk materials $^{32,33}$ but less on surfaces. ${ }^{34-42}$ Recently, Woisel's group has investigated the feasibility of interfacial DA reaction (333 K, 7 days) between maleimide derivatives and furan derivatives on a $\mathrm{TiO}_{2}$ surface grafted with functional polydopamine. The wettability of the surfaces could be tailored by changing the hydrophobicity of the grafted derivatives. ${ }^{43}$ Interfacial DA reaction has also been explored to obtain self-healable composites at relatively low temperatures by performing DA reaction between maleimide derivatives immobilized on fibers ${ }^{44}$ or nanoparticles ${ }^{45}$ and furan-functionalized matrixes. These studies highlighted the necessary control of the DA reactivity to carry out the reaction at a relatively low temperature. The investigation of the DA reactivity for the furan/maleimide pair is well discussed in the literature when performed in solution close to room temperature. $^{46,47}$ These studies reported on the influence of maleimide and furan derivatives on the DA reactivity: the diene should have electrodonor substituents and the dienophile should have electroattractive substituents to lower the energetic gap between the electronic orbitals of the diene and the dienophile and, thus, to decrease the reaction temperature and time. In 2005, our team carried out a comparative study between the interfacial DA reactivity on SAMs and plasma polymers, both containing alkene groups. ${ }^{48}$ The investigation of the DA reactivity of these surfaces with cyclopentadiene showed that the plasma polymer thin film was less reactive than the monolayer but the transition-state complex was more ordered. The chemical environment of the reactants thus controls their reactivity. But what about the effect of the plasma polymer properties on the DA reactivity? Indeed, regarding the operating conditions of low-pressure (pulsed) plasma polymerization, the retention rate of functional groups and the polymer cross-linking rate may change. Steric hindrance and mobility of reactive groups may therefore be modified, impacting the formation of the transition-state complex during DA reaction. In this study, we investigated the influence of plasma polymer properties on the reactivity of the DA reaction between furan groups (diene) within the polymer thin film and maleic anhydride (MA) (dienophile) in water. A simple surface characterization method of the functional coating, based on contact angle measurements, has enabled the calculation of the interfacial conversion of the DA reaction on plasma polymers. These results have led to the suggestion of a reactivity model according to the values of thermodynamic parameters required for the formation of the transition-state complex.

\section{EXPERIMENTAL SECTION}

Materials. Single-side polished silicon wafers $(20 \mathrm{~mm} \times 10 \mathrm{~mm}$, Silicon Materials Inc.) were pretreated by $30 \mathrm{~min}$ UV/ozone cleaning (Nanosciences, ProCleaner 220), followed by $1 \mathrm{~h}$ silanization with 1.9 $\times 10^{-3} \mathrm{~mol} \mathrm{~L}^{-1}$ 1-triethoxysilylprop-2-en-1-amine (92\%, abcr GmbH) in chloroform (99\%, Carlo Erba) at room temperature. Silanized wafers were thoroughly rinsed with chloroform and ethanol and dried under nitrogen flow. Gold wafers were prepared by successive thermal evaporation under high vacuum of $10 \mathrm{~nm}$ chromium and $20 \mathrm{~nm}$ gold on native single-side-polished silicon wafers. Before coating them with a plasma polymer thin film, they were cleaned by $30 \mathrm{~min}$ UV/ozone cleaning. Maleic anhydride (99\%, Aldrich), ground into a fine powder, and furfurylamine (99\%, Alfa Aesar) were used as received. Both precursors were separately loaded into a stoppered glass gas-delivery tube with two compartments and degassed by three freeze-pumpthaw cycles.

Fabrication of Furan-Functionalized Thin Films Assisted by Plasma Polymerization. As described elsewhere, ${ }^{29,48}$ plasma polymerization experiments were carried out in an electrodeless cylindrical glass reactor $\left(6 \mathrm{~cm}\right.$ diameter, $680 \mathrm{~cm}^{3}$ volume, and base pressure $5 \times 10^{-4} \mathrm{mbar}$ ) enclosed in a Faraday cage. The reactor was equipped with a gas inlet on one side and a Pirani pressure gauge and a two-stage rotary pump (Edwards) connected to a liquid nitrogen cold trap on the other side. All joints were grease-free. An externally wound copper coil (4 mm diameter, four turns) was connected to an impedance matching network (Dressler, VM $1500 \mathrm{~W}$-ICP), used to match the output impedance of a $13.56 \mathrm{MHz}$ radio frequency (RF) power supply (Dressler, Cesar 133) to the ionized gas load by minimizing the standing wave ratio of the transmitted power.

Prior to each experiment, the reactor was cleaned by a $30 \mathrm{~min}, 40$ $\mathrm{W}$ air plasma treatment. The system was then vented to air, and the substrates were placed at the center of the chamber $(19 \mathrm{~cm}$ from the chamber inlet), followed by evacuation back down to base pressure. Subsequently, maleic anhydride (MA) vapor was introduced into the reaction chamber at a flow rate of approximately $1 \times 10^{-4} \mathrm{~g} \mathrm{~s}^{-1}$ and a work pressure of $2 \times 10^{-1} \mathrm{mbar}$. At this stage, the plasma was ignited by supplying an average continuous-wave power of $10 \mathrm{~W}$. The power delivered by the RF generator was continuous for $8 \mathrm{~min}$ or pulsed at a frequency of $816 \mathrm{~Hz}$ and a given duty cycle (DC) (eq 1) for $30 \mathrm{~min}$ $(\mathrm{DC}=2 \%)$ or $15 \mathrm{~min}(\mathrm{DC}=20 \%)$ (the reaction times were chosen to obtain similar thicknesses of coating).

$$
\mathrm{DC}=\frac{t_{\mathrm{on}}}{t_{\mathrm{on}}+t_{\mathrm{off}}}=X \%
$$

The on-time $\left(t_{\mathrm{on}}\right)$ generates active sites in the gas phase and at the surface of the substrate, whereas conventional polymerization mainly occurs during the off-time $\left(t_{\text {off }}\right)$.

Upon completion of deposition of poly(maleic anhydride), the RF generator was switched off and the monomer feed was allowed to continue to flow through the system for a further 2 min period before evacuating the reactor back down to base pressure. Then, the reactor was fed with furfurylamine vapor at a pressure of 2 mbar without pumping. Furfurylamine reacted with poly(maleic anhydride) by vapor-phase aminolysis for $5 \mathrm{~min}$. The reactor was finally pumped back down to base pressure before venting it up to atmosphere. The furan-functionalized surfaces (FUR $X \%, X \%$ corresponding to the DC used during plasma polymerization of poly(maleic anhydride)) were removed from the reactor and placed in an oven under vacuum at 393 $\mathrm{K}$ for $1 \mathrm{~h}$ to transform the amide groups, produced by the aminolysis reaction between anhydride groups of the plasma polymer and the amine of furfurylamine compound, into more stable cyclic imide groups. ${ }^{47}$

Diels-Alder Reaction between the Furan-Functionalized Plasma Polymers and Maleic Anhydride. The furan-functionalized surfaces, FUR $X \%$ (diene-functionalized surfaces), were immersed in deionized water at room temperature for $1 \mathrm{~h}$ to remove any precursor molecules and low-molecular-weight chains that were not covalently bonded to the plasma polymer films. They were then dried under nitrogen flow and immersed in a maleic anhydride solution (dienophile solution) at a given concentration, at a given temperature, for a given time to carry out the Diels-Alder reaction. Afterward, the surfaces were rinsed five times with deionized water and dried under nitrogen flow.

Surface Analyses. Polarization-Modulation Infrared Reflection-Absorption Spectroscopy (PM-IRRAS). PM-IRRAS spectra on functionalized gold wafers were recorded with a Bruker IFS66/S Fourier transform infrared spectrometer equipped with a PMA 37 polarization modulation system and a nitrogen-cooled MCT detector. The infrared beam was first p-polarized with a $\mathrm{ZnSe}$ wire grid polarizer (Specac) before passing through a photoelastic modulator 
a<smiles>O=C1C=CC(=O)O1</smiles>
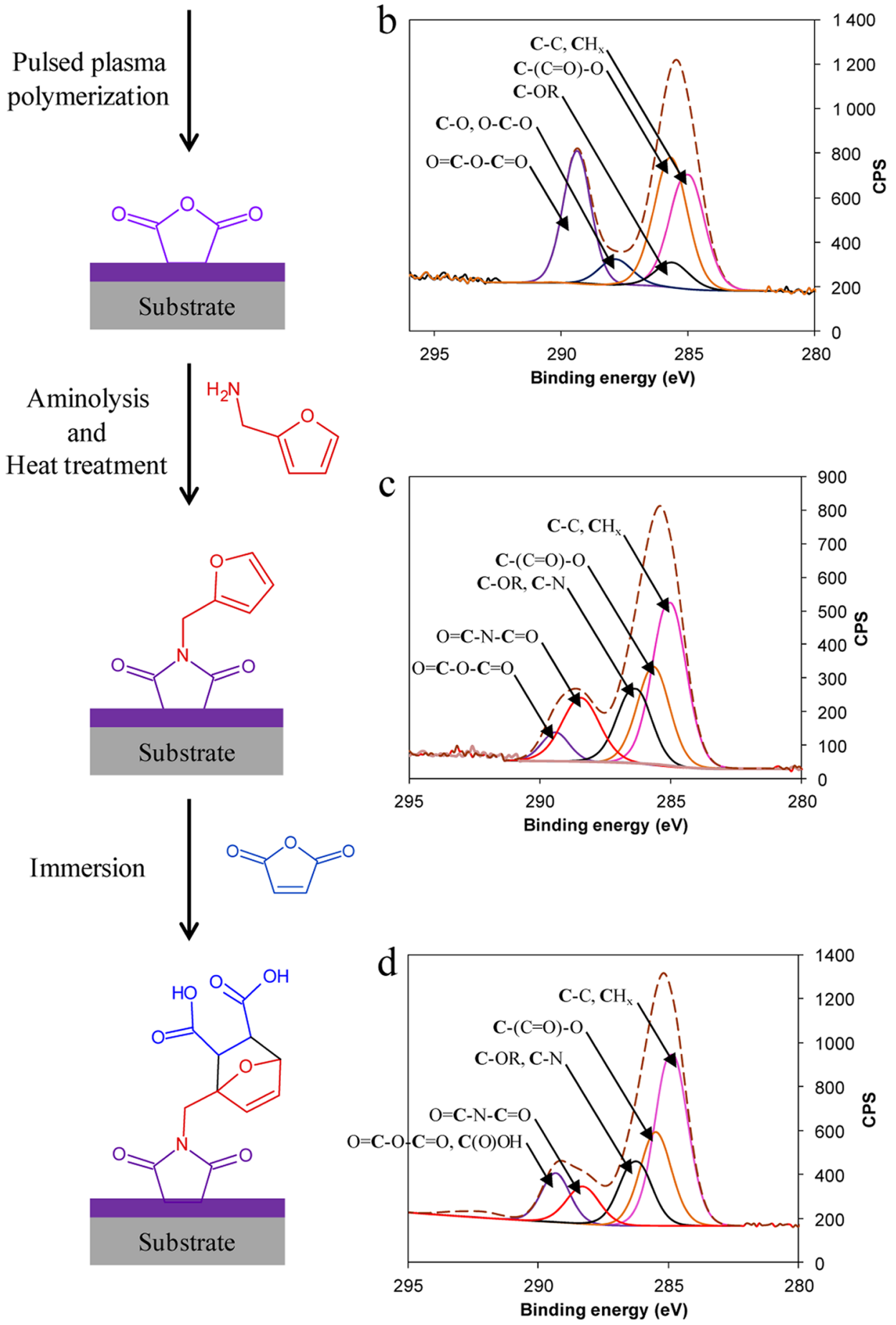

Figure 1. (a) Schematic illustration of the different functionalization steps, and high-resolution XPS spectra of C 1s of the surfaces: (b) after the deposition of the MAPP, (c) after vapor aminolysis (at $293 \mathrm{~K}$ for $5 \mathrm{~min}$ ) and thermal imidization (at $393 \mathrm{~K} \mathrm{for} 1 \mathrm{~h}$ ), and (d) after immersion in an MA aqueous solution ( $303 \mathrm{~K}$ for $5 \mathrm{~h}$ ) leading to the formation of the Diels-Alder adduct between furan-functionalized surfaces and MA.

(PEM-90, Hinds Instruments). A lock-in amplifier (Stanford model SR-830) was used to record the PM-IRRAS spectra. The half-wave retardation frequency was set at $4000 \mathrm{~cm}^{-1}$. A total of 200 scans at a resolution of $4 \mathrm{~cm}^{-1}$ were collected for each measurement at an angle of incidence of $80^{\circ}$ with respect to the normal to the sample surface.

$X$-ray Photoelectron Spectroscopy (XPS). XPS was performed with a VG SCIENTA SES-2002 spectrometer equipped with a concentric hemispherical analyzer. The incident radiation used was generated by a monochromatic $\mathrm{Al} \mathrm{K} \alpha \mathrm{X}$-ray source $(1486.6 \mathrm{eV})$ operating at $420 \mathrm{~W}$ $(14 \mathrm{kV}, 30 \mathrm{~mA})$. Photoemitted electrons were collected at a take-off angle of $90^{\circ}$ from the surface substrate, with electron detection in the constant analyzer energy mode (fixed analyzer transmission). Wide scan spectrum (survey) signals were recorded with a pass energy of $500 \mathrm{eV}$, and for high-resolution spectra ( $\mathrm{C} 1 \mathrm{~s}, \mathrm{O} 1 \mathrm{~s}$, and $\mathrm{N} \mathrm{1s}$ ), the 
pass energy was set to $100 \mathrm{eV}$. The analyzed surface area was approximately $24 \mathrm{~mm}^{2}$, and the base pressure in the analysis chamber during experimentation was about $10^{-9}$ mbar. The spectrometer energy scale was calibrated using the $\mathrm{Ag} 3 \mathrm{~d}_{5 / 2}, \mathrm{Au} 4 \mathrm{f}_{7 / 2}$, and $\mathrm{Cu} 2 \mathrm{p}_{3 / 2}$ core level peaks, set at binding energies of 368.2, 84.0, and $932.6 \mathrm{eV}$, respectively. Spectra were subjected to a Shirley baseline, and peak fitting was made with mixed Gaussian-Lorentzian components with equal full width at half-maximum using CASAXPS version 2.3.18 software. The surface composition expressed in atom $\%$ was determined using integrated peak areas of each component and taking into account the transmission factor of the spectrometer, mean free path (around $30 \AA$ ), and Scofield sensitivity factors of each atom (C 1s: 1.00, O 1s: 2.93, and $\mathrm{N}$ 1s: 1.80). All of the binding energies are referenced to the $\mathrm{C} 1 \mathrm{~s}\left(\mathrm{CH}_{x}\right)$ peak at $285.0 \mathrm{eV}$.

Dynamic Contact Angle (DCA) Measurements. DCA measurements were carried out using a Drop Shape Analyzer (DSA100, Krüss). A droplet of $2 \mu \mathrm{L}$ of buffer solution was cautiously deposited on the surface, and a continuous enlargement of the drop volume was performed at $10 \mu \mathrm{L} \mathrm{min}{ }^{-1}$. Measurements were made on both sides of the drop. The contact angle value obtained just before the meniscus moved corresponded to the indicated advancing contact angle (ACA). Each ACA value was determined by the calculation of the average value of 10 independent measurements. The standard deviation for all average values indicated afterward is about $2^{\circ}$.

To investigate the Diels-Alder reaction kinetics, ACA measurements were carried out with a $\mathrm{KOH} / \mathrm{NaOH}$ (Aldrich) buffer solution at $\mathrm{pH} 13$.

Measurements by Atomic Force Microscopy (AFM). The swelling properties of the polymer thin films (deposited on silicon wafer) were investigated by AFM measurements in deionized water using a FlexAFM scanning probe microscope (Nanosurf), running with a Nanosurf C3000 controller (Nanosurf) and equipped with a liquid cell. Silicon cantilevers with a silicon tip coated with gold (HYDRAALL-G-10) were used for measurements in the contact mode. The spring constant of the cantilever was $0.292 \mathrm{~N} \mathrm{~m}^{-1}$. The swelling properties of the films were quantified by monitoring the evolution in real time at room temperature of the thickness of the polymer coating via a scratch, deliberately made with a needle on the coated substrates.

AFM was also used to determine the thickness of the different thin films in air at room temperature in the tapping mode (free amplitude $=500 \mathrm{mV}$, free vibration frequency $=179 \mathrm{kHz}$ ). Silicon cantilevers (spring constant: $13-77 \mathrm{~N} \mathrm{~m}^{-1}$ ) with a silicon tip ACT-50 were used for these measurements.

Data processing and calculation of the thickness of the coating were performed on $2 \mu \mathrm{m} \times 2 \mu \mathrm{m}$ spectra using WSxM 5.0 software (WSxM Solutions).

\section{RESULTS AND DISCUSSION}

Plasma-Assisted Fabrication and Chemical Composition of Furan-Functionalized Polymer Thin Films (FUR 2\%). A smooth plasma polymer of poly(maleic anhydride) (MAPP) with a thickness of $50 \pm 3 \mathrm{~nm}$ was obtained by lowpressure pulsed plasma polymerization performed at a duty cycle DC $=2 \%$. In Figure $1 \mathrm{~b}$, the X-ray photoelectron spectroscopy (XPS) C 1s envelope was peak-fitted and the following characteristic cyclic anhydride bonds were identified: $285.7 \mathrm{eV}$ (carbon simply bonded to an anhydride group; C$\mathrm{C}(\mathrm{O})-\mathrm{O}$ ) and $289.4 \mathrm{eV}$ (carbon from anhydride groups; $\mathrm{O}=$ $\mathrm{C}-\mathrm{O}-\mathrm{C}=\mathrm{O}$ ). The retention rate of anhydride groups within the plasma polymer, $R$, was calculated by dividing the area of the characteristic peaks of anhydride groups by the total area of the C 1s peak. For DC $=2 \%, R=54 \%$, which is an optimized retention rate obtained in this reactor. ${ }^{29}$

Then, the MAPP was postmodified by aminolysis with furfurylamine performed in vapor phase, followed by a heat treatment. This furan-functionalized thin film is called FUR $2 \%$. This postmodification step was confirmed by high- resolution XPS of C 1s (Figure 1c) thanks to the appearance of a bond at $288.4 \mathrm{eV}$ (carbon from imide groups; $\mathrm{O}=\mathrm{C}-\mathrm{N}-$ $\mathrm{C}=\mathrm{O}$ ). It can be noted that some anhydride groups are still present within the furan-functionalized polymer. The XPS results (see also Figure S1 and Table S1) of these two functionalization steps agree with the literature. ${ }^{27,29}$

Diels-Alder Reaction in Water between FuranFunctionalized Surfaces and Maleic Anhydride. The furan-functionalized plasma polymer FUR $2 \%$ contains diene groups able to react by Diels-Alder reaction with a dienophile. To evidence this reactivity, FUR $2 \%$ surfaces were immersed into an aqueous solution of maleic anhydride (MA, $[\mathrm{MA}]=$ $2.35 \times 10^{-4} \mathrm{~mol} \mathrm{~L}^{-1}$ ) at $303 \mathrm{~K}$ for $5 \mathrm{~h}$. A significant increase of the intensity of the peak at $289.4 \mathrm{eV}$, assigned to anhydride groups $(\mathrm{O}=\mathrm{C}-\mathrm{O}-\mathrm{C}=\mathrm{O})$ and carboxylic acid $(\mathrm{C}(\mathrm{O})-\mathrm{OH})$ in the high-resolution XPS spectra of C 1s (Figure 1d), indicates the presence of the DA adduct on the surface. These results validate the functionalization process described here and the feasibility of carrying out a DA reaction at a moderate temperature in water between furan-functionalized surfaces and $\mathrm{MA}$.

XPS characterizations were confirmed by ACA measurements (Figure S2 in the Supporting Information) and PMIRRAS analyses (Figure S3 in the Supporting Information). Indeed, the presence or absence of the DA adduct on the outermost surface leads to a significant change of ACA values at $\mathrm{pH}$ 13. The furan-functionalized plasma polymer (FUR $2 \%$ ) has an ACA of $67 \pm 2^{\circ}$, whereas an ACA of $35 \pm 3^{\circ}$ characterizes the presence of the adduct on the surface due to the formation of hydrophilic carboxylate groups at high $\mathrm{pH}$. Because of the ease and the rapidity of this surface characterization technique, the presence of the adduct is afterward characterized by ACA measurements at $\mathrm{pH} 13$.

Study of the Interfacial Diels-Alder (DA) Reaction between Furan-Functionalized Surfaces and Maleic Anhydride and Determination of the Related Thermodynamic Parameters. Kinetics and Thermodynamic Parameters of Interfacial DA Reaction Determined by Neglecting the Retro-DA Reaction. The progress of the DA reaction between FUR 2\% and MA in water was investigated by measuring the evolution with the reaction time of the ACA at $\mathrm{pH} 13$ of the surface after its immersion in the dienophile (MA) solution for various concentrations of dienophile ranging from $2.35 \times 10^{-5}$ to $2.35 \times 10^{-4} \mathrm{~mol} \mathrm{~L}^{-1}$ (Figure 2a) and various reaction temperatures ranging from 293 to 323 $\mathrm{K}$ (Figure 2b).

As observed in Figure 2, the cycloaddition between furan and MA at the interface depends on the dienophile concentration and on the temperature of the solution.

The adduct content and the residual furan content on the surface can be calculated from ACA measurements using the Cassie equation (eq 2$)^{49}$

$$
\cos \theta a=\chi_{\text {diene }} \cos \theta_{\text {diene }}+\chi_{\text {adduct }} \cos \theta_{\text {adduct }}
$$

with $\chi_{\text {diene }}$ and $\chi_{\text {adduct }}$ corresponding to the surface densities of the residual furan functions and of the DA adduct groups; $\theta a$ being the experimental ACA at any time; and $\theta_{\text {diene }}$ and $\theta_{\text {adduct }}$ being the ACA measured, respectively, before the DA reaction on FUR 2\%, saturated with furan functions $\left(\theta_{\text {diene }}=67^{\circ}\right)$, and after the DA reaction on the surface fully saturated with the DA adduct $\left(\theta_{\text {adduct }}=35^{\circ}\right)$.

Moreover, if $\chi_{\text {diene }}+\chi_{\text {adduct }}=1$, then the Cassie equation leads to the determination of $\chi_{\text {diene }}$ at any time (eq 3) 

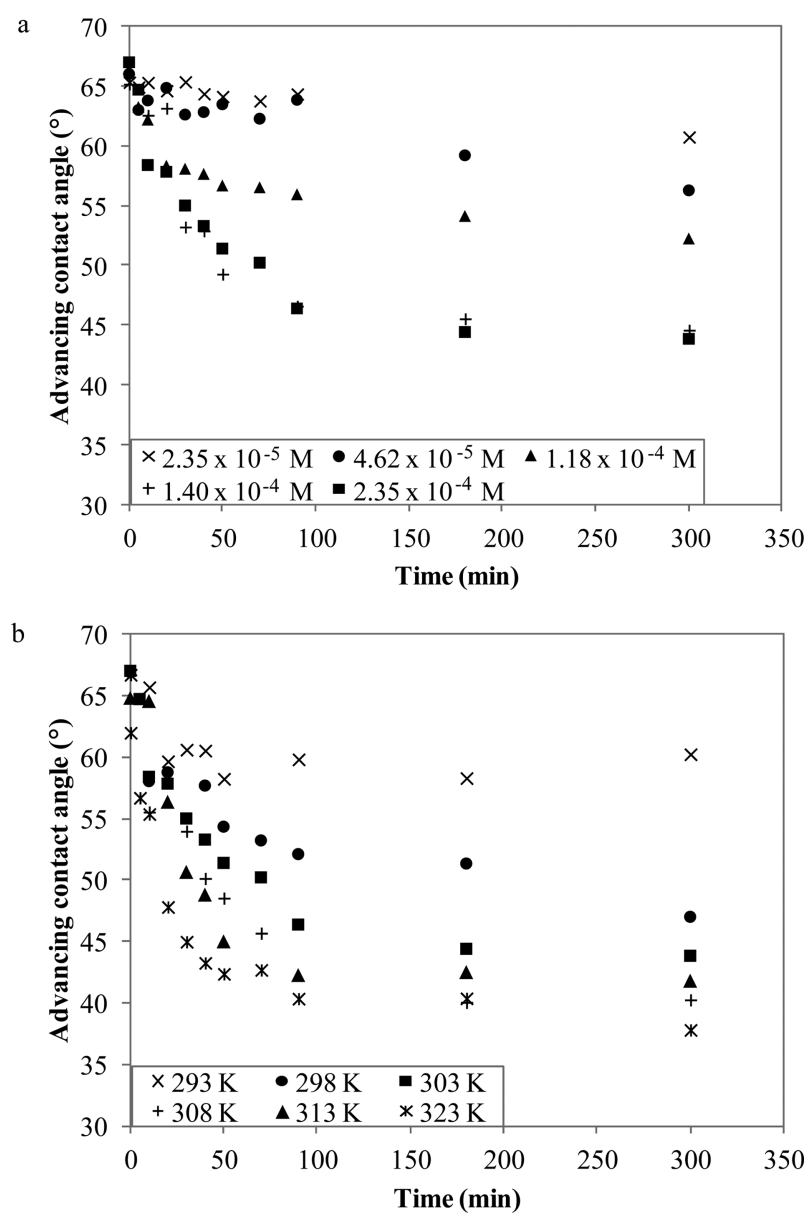

Figure 2. Evolution of the advancing contact angle at $\mathrm{pH} 13$ with immersion time after DA reaction between FUR 2\% and MA in water (a) at $303 \mathrm{~K}$, at different MA concentrations and (b) at different temperatures, at $[\mathrm{MA}]=2.35 \times 10^{-4} \mathrm{~mol} \mathrm{~L}^{-1}$.

$$
\chi_{\text {diene }}=\frac{\cos \theta a-\cos \theta_{\text {adduct }}}{\cos \theta_{\text {diene }}-\cos \theta_{\text {adduct }}}
$$

To better understand this interfacial DA reaction between furan-functionalized surfaces and MA, the rate law of this reaction has first been investigated. By assuming that the reversibility of this interfacial DA reaction can be neglected at the temperatures that were tested, ${ }^{46}$ the reaction rate $\mathrm{v}$ can be expressed as follows (eq 4)

$$
v=k_{\mathrm{DA}}[\mathrm{MA}]^{q}[\mathrm{FUR} 2 \%]^{p}
$$

where $k_{\mathrm{DA}}$ is the DA reaction rate constant, [MA] and [FUR $2 \%$ ] are, respectively, the molar concentration in dienophile and surface content of diene, and $q$ and $p$ represent the partial orders of the reaction related to dienophile and diene, respectively.

To easily determine the partial orders, the amount of dienophile (MA) was introduced in large excess (in solution) compared to the amount of diene groups (on the surface) to be allowed to apply the isolation method. The rate law can thus be simplified (eq 5)

$$
v=k^{\prime}[\text { FUR 2\% }]^{p} \text { with } k^{\prime}=k_{\mathrm{DA}}[\mathrm{MA}]^{q}
$$

In Figure S4 and Table S2 (in the Supporting Information), $\ln \left(\chi_{\text {diene }}\right)$ and $1 / \chi_{\text {diene }}$ were plotted against the reaction time for the different concentrations and the linearity of the graphs was tested. For all concentrations, a linear dependence is observed for $\ln \left(\chi_{\text {diene }}\right)=f($ time $)$ (Table 1$)$. This linear behavior indicates that the DA reaction can be described by pseudofirst-order kinetics related to the diene, therefore $p=1$.

Table 1. Coefficients of Determination $\left(R^{2}\right)$ for the PseudoFirst-Order Kinetics Related to the Diene for FUR 2\% after DA Reaction Carried Out at Different MA Concentrations at $303 \mathrm{~K}$

$\begin{array}{cc}{[\mathrm{MA}]\left(\mathrm{mol} \mathrm{L}^{-1}\right)} & R^{2} \text { (pseudo-first-order kinetics related to the diene) } \\ 2.35 \times 10^{-5} & 0.94 \\ 4.62 \times 10^{-5} & 0.88 \\ 1.18 \times 10^{-4} & 0.92 \\ 1.40 \times 10^{-4} & 0.96 \\ 2.35 \times 10^{-4} & 0.95\end{array}$

Moreover, the slopes of the curves $\ln \left(\chi_{\text {diene }}\right)=f($ time $)$ directly give the values of the observed rate constants $k^{\prime}$ (equal to $\left.k_{\mathrm{DA}}[\mathrm{MA}]^{q}\right)$ for the different concentrations that were tested. In addition to the determination of the value of $k_{\mathrm{DA}}$ at $303 \mathrm{~K}$, the linearization of the curve $\ln \left(k^{\prime}\right)=f(\ln [\mathrm{MA}]$ ) (Figure 3 ) demonstrates that the DA reaction can be described by pseudo-second-order kinetics related to the dienophile since the slope of this curve is close to 2 , therefore $q=2$.

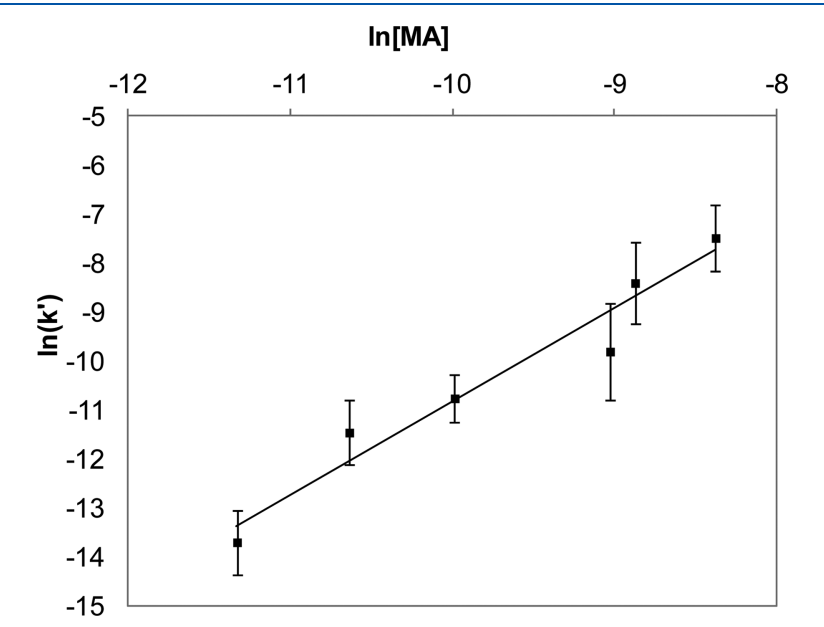

Figure 3. Determination of the partial order related to the dienophile (q) for the DA reaction between FUR 2\% and MA by linearization of the curve $\ln \left(k^{\prime}\right)=f(\ln [\mathrm{MA}])$. The equation of the fitting curve is $\ln \left(k^{\prime}\right)=1.9 \ln ([\mathrm{MA}])+7.9\left(R^{2}=0.95\right)$.

Consequently, the rate law of the DA reaction between furan-functionalized surfaces FUR $2 \%$ and MA can be written as follows (eq 6)

$$
v=\mathrm{k}_{\mathrm{DA}}[\mathrm{MA}]^{2}[\text { FUR 2\%] }
$$

The partial order related to the dienophile (MA), equal to 2 , is quite surprising compared to that in other kinetics studies, especially those performed in solution. ${ }^{47}$ However, a pseudosecond-order kinetics related to the diene or dienophile on surfaces has already been observed in the literature with another diene/dienophile pair. ${ }^{48}$ Two molecules of maleic anhydride, here in solution, seem to be required to interact with one furan group to form the transition-state complex. This cooperative effect may favor the coplanar conformation of 
the transition-state complex involved in this concerted mechanism.

Then, the results of the kinetics conducted at various temperatures enabled the determination of DA rate constants, $k_{\mathrm{DA}}$ at different temperatures (Table 2).

Table 2. Values of Diels-Alder Rate Constants, $k_{\mathrm{DA}}$ Determined at Different Temperatures

$\begin{array}{cc}T(\mathrm{~K}) & k_{\mathrm{DA}} \times 10^{-4}\left(\mathrm{M}^{-2} \mathrm{~s}^{-1}\right) \\ 293 & 5.8 \\ 298 & 9.9 \\ 303 & 22 \\ 308 & 29 \\ 313 & 35 \\ 323 & 65\end{array}$

It can be noted that the values of $k_{\mathrm{DA}}$ increase with the reaction temperature and that they obey the Arrhenius equation (eq 7), as illustrated in Figure 4

$$
k_{\mathrm{DA}}=A \mathrm{e}^{-E_{\mathrm{a}} / R T}
$$

with $A$ the pre-exponential factor related to the frequency of collisions, $E_{\mathrm{a}}$ the activation energy, $T$ the absolute temperature, and $R$ the universal gas constant.

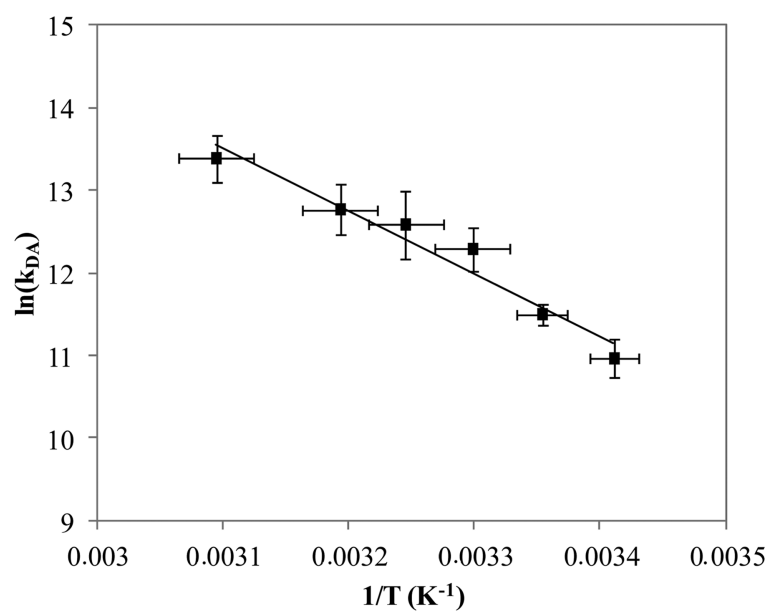

Figure 4. Linearization of the Arrhenius equation representing the evolution of $\ln \left(k_{\mathrm{DA}}\right)$ with $1 / T$. The equation of the fitting curve is $\ln \left(k_{\mathrm{DA}}\right)=-7557 / T+36.9\left(R^{2}=0.95\right)$.

The slope of the linearization plot of the Arrhenius equation gives an estimation of the activation energy of this interfacial DA reaction; here, $E_{\mathrm{a}}=63 \pm 4 \mathrm{~kJ} \mathrm{~mol}^{-1}$, which is the same order of magnitude as another interfacial DA reaction performed on plasma polymer and reported in the literature. ${ }^{48}$

In addition to the determination of the macroscopic activation energy based on collision theory, the values of the rate constant at different temperatures can provide information about the activated complex formed during the reaction by applying transition-state theory. According to the Eyring equation, ${ }^{50}$ the DA rate constant, $k_{\mathrm{DA}}$, can be calculated as written in eq 8

$$
k_{\mathrm{DA}}=\kappa \frac{k_{\mathrm{B}} T}{h} \mathrm{e}^{\Delta S^{\ddagger} / R} \mathrm{e}^{-\Delta H^{\ddagger} / R T}
$$

with $\kappa$ the transmission coefficient that can be considered to be equal to $1 ; R, k_{\mathrm{B}}$, and $h$, respectively, the ideal gas constant, the Boltzmann constant, and Planck's constant, respectively; $T$ the absolute temperature; and $\Delta H^{\neq}$and $\Delta S^{\neq}$, respectively, the enthalpy and entropy of activation related to the formation of the transition-state complex.

By linearization of eq 8 , eq 9 is obtained

$$
\ln \left(\frac{k_{\mathrm{DA}}}{T}\right)=\ln \left(\kappa \frac{k_{\mathrm{B}}}{h}\right)+\frac{\Delta S^{\neq}}{R}-\frac{\Delta H^{\neq}}{R T}
$$

Plotting $\ln \left(k_{\mathrm{DA}} / T\right)=f(1 / T)$ (Figure 5) enables the calculation of thermodynamic parameters of the $\mathrm{DA}$ reaction between

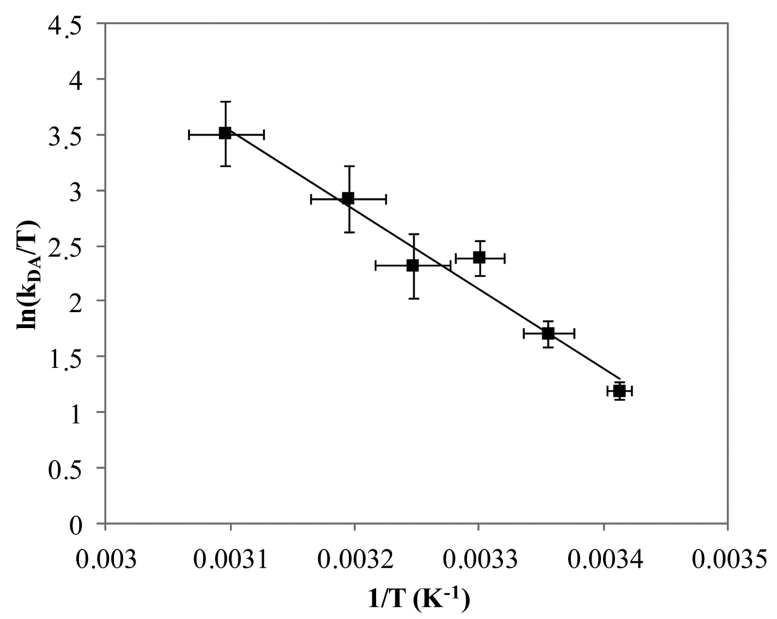

Figure 5. Determination of the enthalpy of activation and entropy of activation by linearization of the Eyring equation. The equation of the fitting curve is $\ln \left(k_{\mathrm{DA}} / T\right)=-7242 / T+30.2\left(R^{2}=0.95\right)$.

FUR 2\% surfaces and MA: the enthalpy of activation of this interfacial DA reaction corresponds to $\Delta H^{\neq}=60 \pm 8 \mathrm{~kJ} \mathrm{~mol}^{-1}$ and the entropy of activation corresponds to $\Delta S^{\neq}=0.05 \pm$ $0.02 \mathrm{~kJ} \mathrm{~mol}^{-1} \mathrm{~K}^{-1}$. Indeed, the slope of this curve is equal to $-\Delta H^{\neq} / R$ and the intercept leads to the determination of $\Delta S^{\neq}$.

$\Delta H^{\neq}$represents the energy needed by the system to form the transition-state complex, and $\Delta S^{\neq}$reflects the order brought by the formation of the activated complex. The positive value of $\Delta H^{\neq}$evidences that the reaction is endothermic. The value of $\Delta S^{\neq}$is very close to 0 , which shows that almost no change of order occurs by the formation of the complex. Although the formation of the transition-state complex induces a coplanar orientation of the diene and dienophile, it is difficult to comment on a more or less ordered system compared to the initial state, at least for the FUR 2\% surface. Indeed, the reactive groups are embedded within the plasma polymer, which has a poorly defined cross-linked structure. Therefore, the order of the system remains almost unchanged during the formation of the transition-state complex. The values of enthalpy and entropy of activation are in accordance with those reported in the literature for interfacial DA reactions $\left(\Delta H^{\neq} \approx 20-70 \mathrm{~kJ} \mathrm{~mol}^{-1}, \Delta S^{\neq} \approx-0.1\right.$ to $\left.-0.5 \mathrm{~kJ} \mathrm{~mol}^{-1} \mathrm{~K}^{-1}\right){ }^{48,51}$ However, we should carefully compare these results because the physicochemical properties (microstructure, cross-linking rate) of the surfaces were different, the solvents used were not the same, and above all, the diene/dienophile pair differed.

Kinetics and Thermodynamic Parameters of Interfacial $D A$ Reaction Determined without Neglecting the Retro-DA 
Reaction. In the previous paragraph, the progress of the retroDA reaction has been neglected in favor of the progress of the DA reaction to simplify the calculations. In this section, we consider the chemical equilibrium of this DA reaction and determine the rate constants of the direct DA reaction, $k_{\mathrm{DA}_{2}}$, and of the retro-cycloaddition, $k_{\mathrm{rDA}_{2}}$, by fitting with MATLAB the evolution of the reaction progress with time.

For this fitting, the rate law, previously determined, changes as follows (eq 10)

$$
v=-\frac{\mathrm{d}[\mathrm{FUR} 2 \%]}{\mathrm{d} t}=k_{\mathrm{DA}_{2}}[\mathrm{MA}]^{2}[\mathrm{FUR} 2 \%]-k_{\mathrm{rDA}_{2}} x
$$

with $x=1-$ [FUR 2\%], and $x$ and [FUR 2\%] being the surface contents of adduct and furan groups, respectively, of the surface solely containing furan and adduct groups.

Since MA was in large excess compared to the amount of furan groups on FUR 2\% surfaces, it is reasonable to say that the concentration of MA remains constant during the reaction, which allows us to define an observed rate constant $k_{2}^{\prime}=$ $k_{\mathrm{DA}_{2}}[\mathrm{MA}]^{2}$.

By solving eqs 10 and 11, describing the theoretical evolution of the reaction progress with time, ${ }^{52}$ can be used to fit the experimental data (Figure S6 in the Supporting Information)

$$
x(t)=\frac{k_{2}^{\prime}}{k_{2}^{\prime}+k_{\mathrm{rDA}}}\left(1-\mathrm{e}^{-\left(k^{\prime 2}+k_{\mathrm{rDA}_{2}}\right) t}\right)
$$

In that way, the DA rate constants, $k_{\mathrm{DA}_{2}}$, the retro-DA rate constants, $k_{\mathrm{rDA}_{2}}$, and the equilibrium reaction constants (eq 12) can be computed at various temperatures and are summarized in Table 3.

$$
K=\frac{k_{\mathrm{DA}_{2}}}{k_{\mathrm{rDA}_{2}}}
$$

Table 3. Values of $k_{\mathrm{DA}_{2}}$ Diels-Alder and $k_{\mathrm{rDA}_{2}}$ Retro-DielsAlder Rate Constants as Well as $K=k_{\mathrm{DA}_{2}} / k_{\mathrm{rDA}_{2}}$ at Different Temperatures Determined by Computing Experimental Results Regarding Equation 11

\begin{tabular}{cccr}
$T(\mathrm{~K})$ & $k_{\mathrm{DA}_{2}} \times 10^{-4}\left(\mathrm{M}^{-2} \mathrm{~s}^{-1}\right)$ & $k_{\mathrm{rDA}}\left(\mathrm{s}^{-1}\right)$ & \multicolumn{1}{c}{$\mathrm{K}$} \\
293 & 2.6 & 706 & 36 \\
298 & 12 & 162 & 740 \\
303 & 21 & 17 & 12600 \\
308 & 21 & 66 & 3200 \\
313 & 25 & 86 & 2920 \\
323 & 60 & 216 & 2790 \\
\hline
\end{tabular}

By comparing the values of the DA rate constants at different temperatures (Tables 2 and 3), it can be noted that $k_{\mathrm{DA}}$ and $k_{\mathrm{DA}_{2}}$ are very close to each other for most of the tested temperatures. Moreover, the retro-DA rate constants, $k_{\mathrm{rDA}_{2}}$, are quite low and the values of the equilibrium reaction constant, $\mathrm{K}$, are high in the tested temperature range. These observations lead to the conclusion that the retro-cycloaddition can be neglected from 293 to $323 \mathrm{~K}$. Therefore, we have decided to ignore the contribution of the retro-DA reaction in the following comparative study of thermodynamic parameters related to the interfacial DA reaction between maleic anhydride in solution and furan-functionalized surfaces with different physicochemical properties.

Influence of the Plasma Polymer Properties on the Interfacial DA Reactivity. Since changing the operating conditions of plasma polymerization modifies the steric hindrance and the mobility of the reactive groups as well as their retention rate, ${ }^{25}$ it is interesting to investigate the impact of these properties on the DA reactivity. For this purpose, different plasma polymers with various cross-linking rates and anhydride contents (but similar thicknesses) were fabricated by varying the plasma duty cycle (DC). Poly(maleic anhydride) (MAPP $X \%, X$ being the plasma DC) was thus synthesized at a DC of 2, 20, and 100\% (continuous plasma). As shown in the XPS spectra in Figure $6 a-c$, the different surfaces have different anhydride retention rates $(R): R=54 \%$ for MAPP $2 \%, R=30 \%$ for MAPP $20 \%$, and $R=22 \%$ for MAPP $100 \%$. Since the aminolysis with furfurylamine is very efficient (as previously illustrated), it can be considered that the evolution of the furan content, after performing the postmodification step on these surfaces, is similar regarding the plasma DC. Moreover, AFM measurements of the thickness of MAPP X\% as a function of the immersion time of the polymer thin film in water enable the estimation of the swelling rates of these plasma polymers (Figure $6 \mathrm{~d}-\mathrm{f}$ ). Swelling rates varying from $10 \%$ to over $70 \%$ can be achieved by changing the plasma polymerization conditions. Indeed, the swelling rate of a plasma polymer is directly related to its cross-linking, which can be tuned by changing the plasma DC.

Consequently, various furan-functionalized surfaces, FUR $X$ $\%$, have been prepared by changing the plasma DC during plasma polymerization of maleic anhydride. As illustrated in Figure 7, surfaces with a high swelling rate and a high retention rate of functional groups were prepared at low plasma DC, whereas surfaces with a high cross-linking rate and few functional groups were obtained by continuous plasma polymerization.

The methodology previously described was applied again to investigate the kinetics and to determine the thermodynamic parameters (activation energy, enthalpy of activation, and entropy of activation) of the interfacial DA reaction between various furan-functionalized surfaces and maleic anhydride. The reaction progress with immersion time was measured at different concentrations and temperatures to see whether the properties of the polymer thin film influence the reactivity of the surfaces regarding DA reaction. First, we verified that the partial orders related to the reactants were the same for the three surfaces, namely, a first order for the diene on the surface and a second order for the dienophile in solution. Second, the rate constants $k_{\mathrm{DA}}$ were determined at various temperatures for the two additional plasma polymers (Table S3 in the Supporting Information). Similarly to FUR 2\%, it can be noted that the DA rate constant increases with temperature. However, the increase with temperature is slower and rate constants are lower when the plasma polymer is more crosslinked. This means that little cross-linking favors a faster DA reaction, which probably originates in the higher probability of collision with reactive groups that are appropriately oriented to undergo the DA reaction. Indeed, the FUR $2 \%$ surface has a higher density of functional groups and the reactive groups are more flexible, so easier to reach. Besides, the activation energy of the reaction performed on the three surfaces significantly differs from 25 to $63 \mathrm{~kJ} \mathrm{~mol}^{-1}$ for FUR $100 \%$ and FUR 2\%, 
a

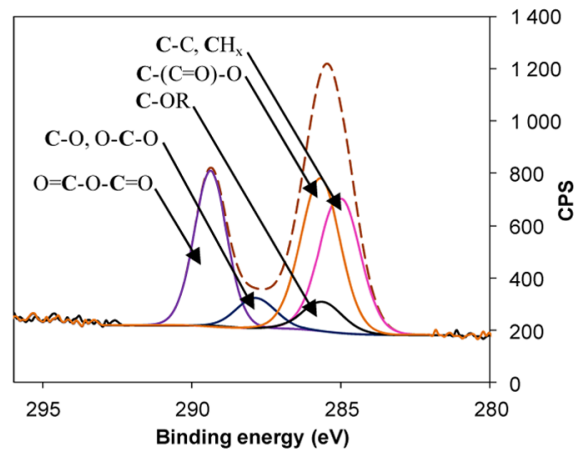

b

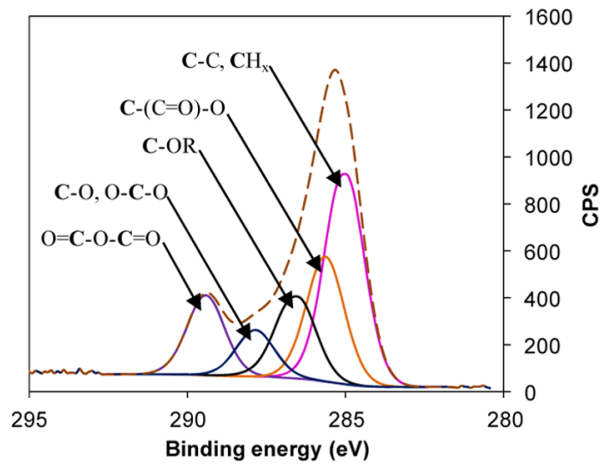

c

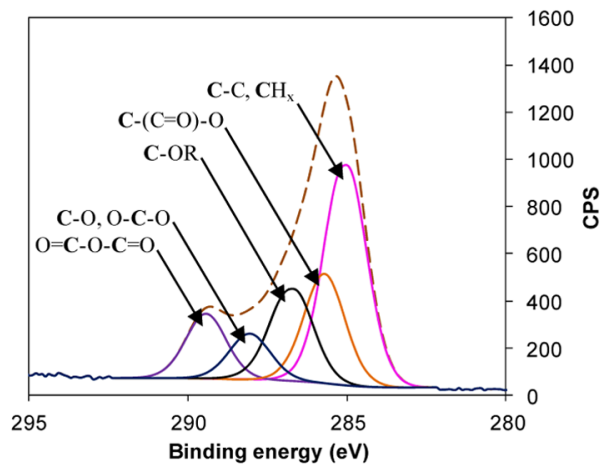

d

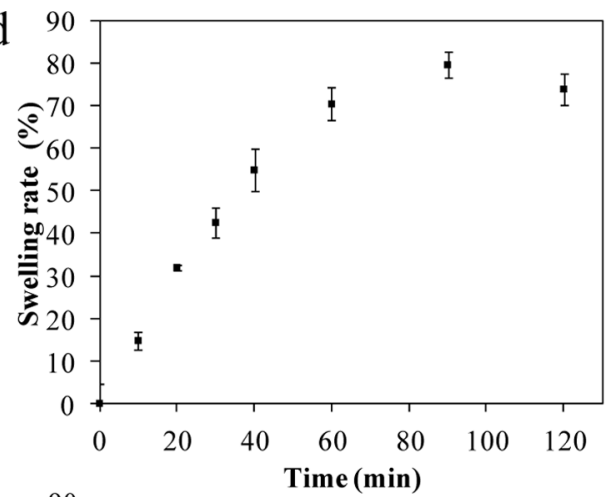

e
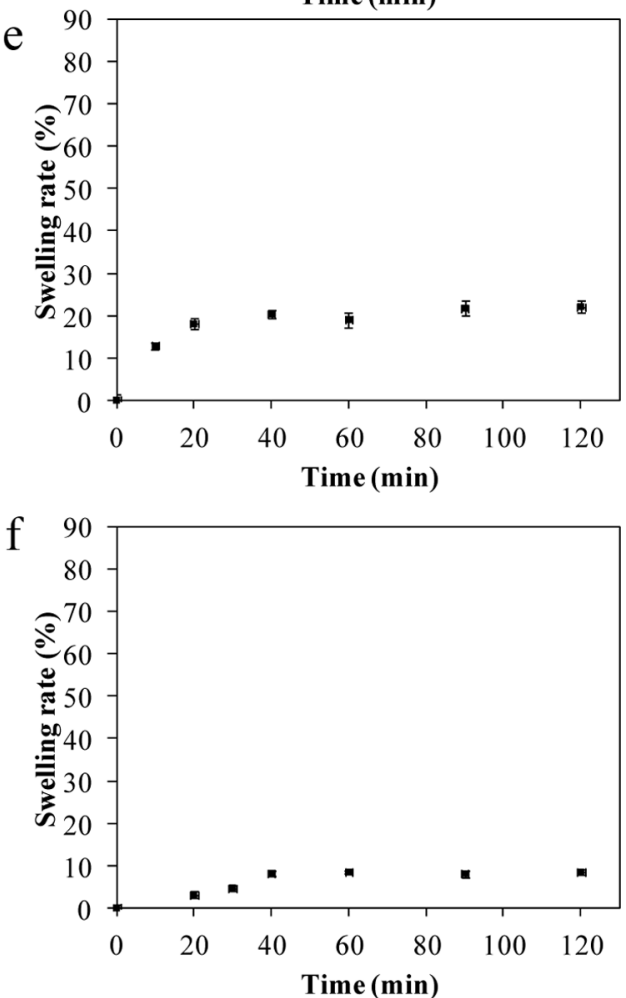

Figure 6. (Left) High-resolution XPS spectra of $\mathrm{C}$ 1s and (right) AFM swelling rates determined by AFM measurements of MAPP synthesized by pulsed plasma polymerization at a DC of (a,d) $2 \%$, (b, e) $20 \%$, and (c, f) $100 \%$.

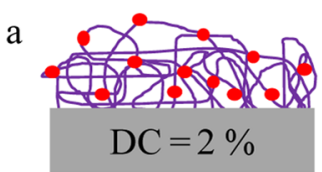

b

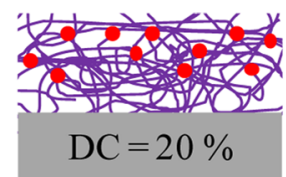

c

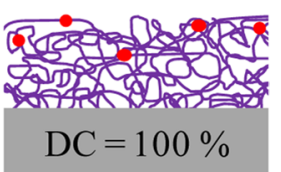

Figure 7. Schemes of the different functional surfaces after pulsed plasma polymerization performed at a DC of (a) $2 \%$, (b) $20 \%$, and (c) $100 \%$. The red points represent the functional groups available for interfacial DA reaction, and the purple lines represent the polymer chains.

respectively. As illustrated in Figure 7, the functional groups are fairly static due to the high cross-linking rate for the FUR $100 \%$ surface. Molecular vibrations are thus hindered by crosslinking. Additionally, the accessible functional groups are those located at the outermost surface. The DA reaction with these functional groups does not therefore require a high activation energy since the latter are rather accessible and stationary. Table 4 summarizes the values of enthalpy of activation, entropy of activation, and free energy of activation at $303 \mathrm{~K}$ regarding the physicochemical properties of the functional surfaces.
Table 4. Values of Thermodynamic Parameters Characterizing the Formation of the Transition-State Complex Involved during the Interfacial DA Reaction for Different Plasma Polymers

\begin{tabular}{lcccc}
\multicolumn{1}{c}{ samples } & $\begin{array}{c}E_{\mathrm{a}} \\
\left.(\mathrm{kJ} \mathrm{mol})^{-1}\right)\end{array}$ & $\begin{array}{c}\Delta H^{\neq} \\
\left(\mathrm{kJ} \mathrm{mol}^{-1}\right)\end{array}$ & $\begin{array}{c}\Delta S^{\neq} \\
\left(\mathrm{kJ} \mathrm{mol}^{-1} \mathrm{~K}^{-1}\right)\end{array}$ & $\begin{array}{c}\Delta G^{\neq a} \\
\left(\mathrm{~kJ} \mathrm{~mol}^{-1}\right)\end{array}$ \\
FUR 2\% & $63 \pm 4$ & $60 \pm 8$ & $0.05 \pm 0.02$ & $44 \pm 8$ \\
FUR 20\% & $40 \pm 1$ & $38 \pm 1$ & $-0.05 \pm 0.01$ & $53 \pm 2$ \\
FUR & $25 \pm 2$ & $22 \pm 2$ & $-0.10 \pm 0.01$ & $53 \pm 2$ \\
$\quad$ 100\% & & & &
\end{tabular}

${ }^{a}$ Calculated at $303 \mathrm{~K}$. 
The increase of the enthalpy of activation to get the transition-state complex when the polymers are less crosslinked can be explained similarly to the increase of activation energy. The formation of the activated complex requires more energy since functional groups that are located deeper within the plasma polymer can be involved in the formation of the complex. Furthermore, the complex may be more difficult to achieve because of the fact that the functional groups are randomly oriented and because of the higher molecular vibrations that may occur within the less cross-linked polymer. As for the entropy of activation, it is interesting to note that slightly negative values have been calculated for more crosslinked polymers. This result is understandable since the interfacial DA reaction probably occurs more favorably at the outermost surface compared to FUR 2\%. Therefore, some order can be gained during the formation of the transition-state complex since the concerted mechanism occurring close to the surface may bring some order to the system. The free energy of activation $\Delta G^{f}$ does not change significantly with the physicochemical properties of the surfaces, which is commonly observed. $^{53}$

These data evidence that differences in the thin-film microstructure imply significant changes in the kinetics and the thermodynamic parameters of the reaction and the formation of the transition-state complex of this interfacial DA reaction. Consequently, it is possible to tune the interfacial reactivity by tailoring the physicochemical properties of the polymer thin film directly via the selected operating conditions of plasma polymerization.

\section{CONCLUSIONS}

In this work, we investigated the interfacial Diels-Alder (DA) reactivity on plasma polymer coatings with different physicochemical properties. First, well-adhering poly(maleic anhydride) thin films were deposited by low-pressure plasma polymerization conducted at different duty cycles (DC), and a subsequent postfunctionalization with a furan-terminated molecule was carried out. The reactivity of these furanfunctionalized surfaces was then quantified by determining the kinetics and thermodynamic parameters of the interfacial DA reaction with MA (in solution). Since the operating conditions of plasma polymerization, in particular the plasma DC, strongly influence the density of functional groups and the polymer cross-linking rate, we have expanded this study to the understanding of the influence of the physicochemical composition of plasma polymer on this interfacial DA reactivity. After identifying the rate law of the reaction (firstorder kinetics related to the diene on the surface and secondorder kinetics related to the dienophile in solution), we have compared the values of DA rate constants and activation parameters to suggest a reactivity model for this interfacial cycloaddition. A plasma polymer with numerous functional groups and low cross-linking rate favors a fast DA reaction, probably due to the higher probability of finding appropriately oriented reactive groups to conduct the cycloaddition. Besides, the activation energy of the reaction performed on such polymers is as high as $63 \mathrm{~kJ} \mathrm{~mol}^{-1}$. This result has been attributed to the high flexibility and mobility of furan groups resulting in their random orientation. For the same reason, the enthalpy of activation to form the transition-state complex is quite high with low-cross-linked polymers and functional groups located deeper into the plasma polymer probably contributing to the surface reactivity. In conclusion, we have shown for the first time that the interfacial DA reactivity (between furan-functionalized surfaces and maleic anhydride in solution) can be tuned by the physicochemical properties of the polymer coating. Therefore, by simply varying the operating conditions of plasma polymerization, it is possible to control an interfacial thermoreversible reaction performed on plasma polymers, which opens innovative perspectives for the design of smart coatings.

\section{ASSOCIATED CONTENT}

\section{S Supporting Information}

The Supporting Information is available free of charge on the ACS Publications website at DOI: 10.1021/acs.langmuir.8b02045.

Additional characterizations of the functional thin films (XPS wide scans, PM-IRRAS spectra, and contact angle measurements); complementary figures and tables with experimental data (PDF)

\section{AUTHOR INFORMATION}

\section{Corresponding Author}

*E-mail: florence.bally-le-gall@uha.fr.

ORCID $\odot$

Fouzia Boulmedais: 0000-0002-4934-9276

Notes

The authors declare no competing financial interest.

\section{ACKNOWLEDGMENTS}

The authors are grateful for the financial support provided by the ANR (National Agency for Research, ANR-15-CE08-000601 ) and the Universite de Haute-Alsace. J.C. Oliveira acknowledges the doctoral scholarship from Elisabeth and Barbara Grammel Foundation at Freiburg University. They thank Marie-Pierre Laborie for fruitful discussions and Philippe Fioux, Simon Gree, Philippe Kunemann, and Aissam Airoudj for their help with surface characterizations and access to the IS2M and ICS characterization platforms.

\section{REFERENCES}

(1) Stuart, M. A. C.; Huck, W. T. S.; Genzer, J.; Müller, M.; Ober, C.; Stamm, M.; Sukhorukov, G. B.; Szleifer, I.; Tsukruk, V. V.; Urban, M.; Winnik, F.; Zauscher, S.; Luzinov, I.; Minko, S. Emerging applications of stimuli-responsive polymer materials. Nat. Mater. 2010, 9, 101-113.

(2) Theato, P.; Sumerlin, B. S.; O’Reilly, R. K.; Epps, T. H. Stimuli responsive materials. Chem. Soc. Rev. 2013, 42, 7055-7056.

(3) De las Heras Alarcon, C.; Pennadam, S.; Alexander, C. Stimuli responsive polymers for biomedical applications. Chem. Soc. Rev. 2005, 35, 276-285.

(4) Kasemo, B. Biological surface science. Surf. Sci. 2002, 500, 656677.

(5) Guney, A.; Kara, F.; Ozgen, O.; Aksoy, E. A.; Hasirci, V.; Hasirci, N. Surface Modification of Polymeric Biomaterials. In Biomaterials Surface Science; Taubert, A., Mano, J. F., Rodriguez-Cabello, J. C., Eds.; Wiley-VCH, 2013; pp 89-158.

(6) Bally, F.; Ross, A. M. Functionalized Surfaces: Biomolecular Surface Modification with Functional Polymers. In Encyclopedia of Biomedical Polymers and Polymeric Biomaterials; Mishra, M., Ed.; Taylor and Francis, 2016; pp 585-615.

(7) Ulman, A. Formation and structure of self-assembled monolayers. Chem. Rev. 1996, 96, 1533-1554.

(8) Richert, L.; Lavalle, P.; Payan, E.; Shu, X. Z.; Prestwich, G. D.; Stoltz, J. F.; Schaaf, P.; Voegel, J. C.; Picart, C. Layer by layer buildup 
of polysaccharide films: physical chemistry and cellular adhesion aspects. Langmuir 2004, 20, 448-458.

(9) Decher, G. Fuzzy nanoassemblies: toward layered polymeric multicomposites. Science 1997, 277, 1232-1237.

(10) Schaaf, P.; Voegel, J. C.; Jierry, L.; Boulmedais, F. Sprayassisted polyelectrolyte multilayer buildup: From step-by-step to single-step polyelectrolyte film constructions. Adv. Mater. 2012, 24, $1001-1016$.

(11) Dugas, V.; Chevalier, Y. Surface hydroxylation and silane grafting on fumed and thermal silica. J. Colloid Interface Sci. 2003, 264, 354-361.

(12) Wu, Z.; Pittman, C. U., Jr; Gardner, S. D. Grafting isocyanateterminated elastomers onto the surfaces of carbon fibers: reaction of isocyanate with acidic surface functions. Carbon 1996, 34, 59-67.

(13) Bhattacharya, A. Grafting: a versatile means to modify polymers: techniques, factors and applications. Prog. Polym. Sci. 2004, 29, 767-814.

(14) Faure, E.; Falentin-Daudré, C.; Jérôme, C.; Lyskawa, J.; Fournier, D.; Woisel, P.; Detrembleur, C. Catechols as versatile platforms in polymer chemistry. Prog. Polym. Sci. 2013, 38, 236-270.

(15) Barclay, T. G.; Hegab, H. M.; Clarke, S. R.; Ginic-Markovic, M. Versatile Surface Modification Using Polydopamine and Related Polycatecholamines: Chemistry, Structure, and Applications. Adv. Mater. Interfaces 2017, 4, No. 1601192.

(16) Jones, A. C.; Hitchman, M. L. Chemical Vapor Deposition: Precursors, Processes and Applications; Royal Society of Chemistry, 2009.

(17) Siffer, F.; Ponche, A.; Fioux, P.; Schultz, J.; Roucoules, V. A chemometric investigation of the effect of the process parameters during maleic anhydride pulsed plasma polymerization. Anal. Chim. Acta 2005, 539, 289-299.

(18) Choukourov, A.; Biederman, H.; Kholodkov, I.; Slavinska, D.; Trchova, M.; Hollander, A. Properties of amine-containing coatings prepared by plasma polymerization. J. Appl. Polym. Sci. 2004, 92, 979-990.

(19) Airoudj, A.; Debarnot, D.; Beche, B.; Poncin-Epaillard, F. New sensitive layer based on pulsed plasma-polymerized aniline for integrated optical ammonia sensor. Anal. Chim. Acta 2008, 626, $44-52$.

(20) Rinsch, C. L.; Chen, X.; Panchalingam, V.; Eberhart, R. C.; Wang, J. H.; Timmons, R. B. Pulsed radio frequency plasma polymerization of allyl alcohol: Controlled deposition of surface hydroxyl groups. Langmuir 1996, 12, 2995-3002.

(21) Cicala, G.; Milella, A.; Palumbo, F.; Rossini, P.; Favia, P.; d'Agostino, R. Nanostructure and composition control of fluorocarbon films from modulated tetrafluoroethylene plasmas. Macromolecules 2002, 35, 8920-8922.

(22) Chahine, C.; Poncin-Epaillard, F.; Debarnot, D. Plasma copolymerization of fluorinated and acrylate monomers: kinetics and chemical structure study. Plasma Process. Polym. 2015, 12, 493501.

(23) Friedrich, J. Mechanisms of plasma polymerization-reviewed from a chemical point of view. Plasma Process. Polym. 2011, 8, 783802.

(24) Yasuda, H. Plasma Polymerization; Academic Press, 1985.

(25) Veuillet, M.; Ploux, L.; Airoudj, A.; Gourbeyre, Y.; GaudichetMaurin, E.; Roucoules, V. Macroscopic control of DMAHEMA and HEMA plasma polymerization to tune the surface mechanical properties of hydrogel-like coatings. Plasma Process. Polym. 2017, 14, No. 1600215.

(26) Hegemann, D.; Hossain, M. M.; Körner, E.; Balazs, D. J. Macroscopic description of plasma polymerization. Plasma Process. Polym. 2007, 4, 229-238.

(27) Ryan, M. E.; Hynes, A. M.; Badyal, J. P. S. Pulsed plasma polymerization of maleic anhydride. Chem. Mater. 1996, 8, 37-42.

(28) Schiller, S.; Hu, J.; Jenkins, A. T. A.; Timmons, R. B.; SanchezEstrada, F. S.; Knoll, W.; Förch, R. Chemical structure and properties of plasma-polymerized maleic anhydride films. Chem. Mater. 2002, 14, $235-242$.
(29) Airoudj, A.; Schrodj, G.; Vallat, M. F.; Fioux, P.; Roucoules, V. Influence of plasma duty cycle during plasma polymerization in adhesive bonding. Int. J. Adhes. Adhes. 2011, 31, 498-506.

(30) Diels, O.; Alder, K. Synthesen in der hydroaromatischen Reihe. Justus Liebigs Ann. Chem. 1928, 460, 98-122.

(31) Tasdelen, M. A. Diels-Alder "click" reactions: recent applications in polymer and material science. Polym. Chem. 2011, 2, $2133-2145$.

(32) Canadell, J.; Fischer, H.; De With, G.; Van Benthem, R. A. T. M. Stereoisomeric effects in thermo-remendable polymer networks based on Diels-Alder crosslink reactions. J. Polym. Sci., Part A: Polym. Chem. 2010, 48, 3456-3467.

(33) Gandini, A. The furan/maleimide Diels-Alder reaction: a versatile click-unclick tool in macromolecular synthesis. Prog. Polym. Sci. 2013, 38, 1-29.

(34) Dirlam, P. T.; Strange, G. A.; Orlicki, J. A.; Wetzel, E. D.; Costanzo, P. J. Controlling surface energy and wetability with DielsAlder chemistry. Langmuir 2010, 26, 3942-3948.

(35) Peterson, A. M.; Jensen, R. E.; Palmese, G. R. Thermoreversible and remendable glass-polymer interface for fiber-reinforced composites. Compos. Sci. Technol. 2011, 71, 586-592.

(36) Tarducci, C.; Badyal, J. P. S.; Brewer, S. A.; Willis, C. DielsAlder chemistry at furan ring functionalized solid surfaces. Chem. Commun. 2005, 406-408.

(37) Roucoules, V.; Fail, C. A.; Schofield, W. C. E.; Teare, D. O. H.; Badyal, J. P. S. Diels- Alder Chemistry on Alkene Functionalized Films. Langmuir 2005, 21, 1412-1415.

(38) Chen, G.; Gupta, M.; Chan, K.; Gleason, K. K. Initiated chemical vapor deposition of poly (furfuryl methacrylate). Macromol. Rapid Commun. 2007, 28, 2205-2209.

(39) Moreno-Couranjou, M.; Manakhov, A.; Boscher, N. D.; Pireaux, J. J.; Choquet, P. A Novel Dry Chemical Path Way for Diene and Dienophile Surface Functionalization toward Thermally Responsive Metal-Polymer Adhesion. ACS Appl. Mater. Interfaces 2013, 5, 8446-8456.

(40) Aragonès, A. C.; Haworth, N. L.; Darwish, N.; Ciampi, S.; Bloomfield, N. J.; Wallace, G. G.; Diez-Perez, I.; Coote, M. L. Electrostatic catalysis of a Diels-Alder reaction. Nature 2016, 531, $88-91$.

(41) Preuss, C. M.; Goldmann, A. S.; Trouillet, V.; Walther, A.; Barner-Kowollik, C. Biomimetic Dopamine-Diels-Alder Switches. Macromol. Rapid Commun. 2013, 34, 640-644.

(42) Houseman, B. T.; Huh, J. H.; Kron, S. J.; Mrksich, M. Peptide chips for the quantitative evaluation of protein kinase activity. Nat. Biotechnol. 2002, 20, 270-274.

(43) Laure, W.; Woisel, P.; Lyskawa, J. Switching the wettability of titanium surfaces through diels-alder chemistry. Chem. Mater. 2014, 26, 3771-3780.

(44) Zhang, W.; Duchet, J.; Gérard, J. F. Self-healable interfaces based on thermo-reversible Diels-Alder reactions in carbon fiber reinforced composites. J. Colloid Interface Sci. 2014, 430, 61-68.

(45) Engel, T.; Kickelbick, G. Thermoreversible reactions on inorganic nanoparticle surfaces: Diels-alder reactions on sterically crowded surfaces. Chem. Mater. 2013, 25, 149-157.

(46) Gandini, A.; Coelho, D.; Silvestre, A. J. D. Reversible click chemistry at the service of macromolecular materials. Part 1: Kinetics of the Diels-Alder reaction applied to furan-maleimide model compounds and linear polymerizations. Eur. Polym. J. 2008, 44, 4029-4036.

(47) Rulíšek, L.; Sebek, P.; Havlas, Z.; Čapek, P.; Svatoš, A. An experimental and theoretical study of stereoselectivity of furan-maleic anhydride and furan-maleimide Diels-Alder reactions. J. Org. Chem. 2005, 70, 6295-6302.

(48) Siffer, F.; Schultz, J.; Roucoules, V. Alkene Pulsed Plasma Functionalized Surfaces: An Interfacial Diels-Alder Reaction Study. In Adhesion: Current Research and Applications; Possart, W., Ed.; WileyVCH, 2011; pp 289-303.

(49) Cassie, A. B. D. Contact angles. Discuss. Faraday Soc. 1948, 3, $11-16$. 
(50) Eyring, $\mathrm{H}$. The Activated Complex and the Absolute Rate of Chemical Reactions. Chem. Rev. 1935, 17, 65-77.

(51) Kwon, Y.; Mrksich, M. Dependence of the rate of an interfacial Diels-Alder reaction on the steric environment of the immobilized dienophile: an example of enthalpy-entropy compensation. J. Am. Chem. Soc. 2002, 124, 806-812.

(52) Benson, S. W. The Foundations of Chemical Kinetics; McGrawHill, 1960.

(53) Liu, L.; Guo, Q. X. Isokinetic relationship, isoequilibrium relationship, and enthalpy-entropy compensation. Chem. Rev. 2001, 101, 673-696. 